\title{
Kompakte Gleichstromquelle in Schaltnetzteil-Technologie
}

Dlati lating electronic GmbH präsentiert zur Hannover Messe eine ultra-kompakte 12-kWGleichstromquelle (Power Station pe4606-2). Die neue Gleichstromquelle basiert auf dem bewährten 5-kW-Modell Power Station pe4606 in 19"-Einschubkassettentechnik und deckt mit digitaler Regelung den Leistungsbereich von $6 \mathrm{~kW}$ bis $12 \mathrm{~kW}$ und 600 Ausgangsstrom mit einer maximalen Ausgangsspannung bis $100 \mathrm{~V}$ ab. Aufgrund der kompakten Bauweise in Verbindung mit dem breiten Leistungsspektrum kann diese Stromquelle universell eingesetzt werden, beispielsweise als Stand-alone Station

\section{Energieeffiziente Pumpengeneration}

Cager + Mack präsentiert auf dem ZZVO-Gemeinschaftsstand eine neue, hocheffiziente Pumpengeneration. Ausgerüstet mit den neuen IE4Premium-Efficiency-Motoren erzielen sie in den unterschiedlichen Pumpengrößen Energieeinsparungen von bis zu 50 \% gegenüber konventionellen Pumpen. Die durchschnittliche Amortisationszeit beträgt nach Angaben des Unternehmens nur wenige Monate. Bei den neuen permanenterregten Antriebsmotoren (IE4) lässt sich durch die Drehzahlregelung des Frequenzumformers der genaue Betriebspunkt sicher einstellen, was den Energieverbrauch deutlich senkt.

Tel. 07904 / 97 15-0, www.sager-mack.com Halle 3, Stand 54(1)

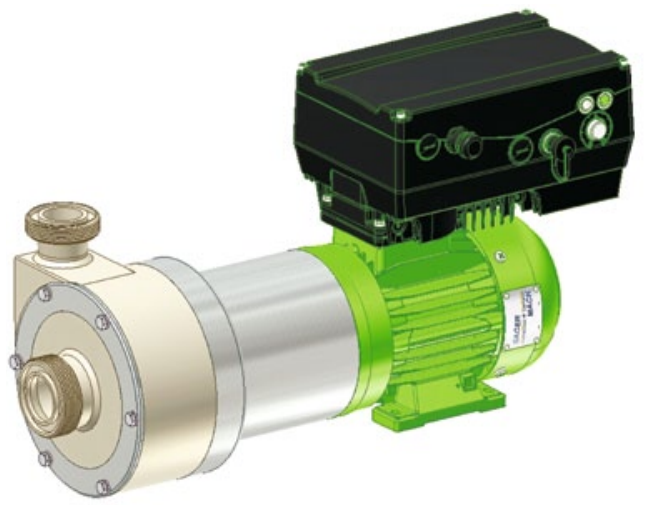

oder im Schaltschrank. Weitere Vorzüge dieser Gleichstromquelle sind unter anderem die geringe Restwelligkeit von $<1 \%$ sowie die genaue, digitale Regelung und der hohe energiesparende Wirkungsgrad. Das Edelstahlgehäuse mit optimierter, neuartiger Kühlluftführung und Lüftersteuerung zum Schutz des Gleichrichters und seiner Elektronik sorgt für eine längere Lebensdauer des Gerätes im Vergleich zu Stromquellen mit konventioneller Luftkühlung. Ein möglicher Austausch des Ventilators zu einem späteren Zeitpunkt kann durch wenige Handgriffe schnell und einfach ausgeführt werden. Die Stromquel- le eignet sich für den Einsatz in den typischen galvanotechnischen Anwendungen, wie zum Beispiel Edelmetallgalvanik, Band- und Handanlagen sowie Leiterplattenanlagen. Tel. 07666 9009-0, www.plating.de Halle 3, Stand E 45

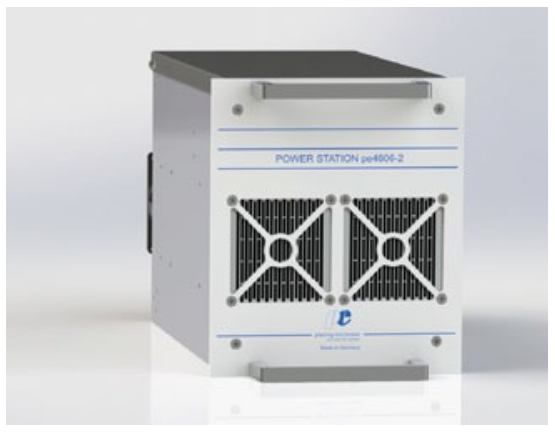

\section{Zuverlässiges kontinuierliches Niveaumesssystem}

$\mathrm{D}$ ie Mazurczak Elektrowärme $\mathrm{GmbH}$ erweitert durch ein kontinuierliches Niveaumesssystem ihren Produktbereich der Füllstandsmesstechnik. Das neue Produkt wird erstmals auf der Hannover Messe ausgestellt. Das kontinuierliche $\mathrm{Ni}$ veaumesssystem KNS erfasst Füllstände unterschiedlicher aggressiver Flüssigkeiten und Wässer. Es wandelt diese in ein 4...20 mA-Signal um. Dadurch können Füllstände in einem Behälter kontinuierlich gemessen und beispielsweise über eine SPS ausgewertet werden. Zudem steht ein innerhalb des Messbereichs frei wählbarer Grenzwert als Transistor-Schaltausgang zur Verfügung. Ein Vorteil des hier eingesetzten, sehr schnellen TDR-Messverfahrens ist, dass das Messergebnis kaum durch die Eigenschaften des zu messenden Mediums wie etwa Dichte, Leitfähigkeit und Dielektrizitätskonstante oder durch die Umgebungsbedingungen wie etwa Druck und Temperatur beeinflusst wird. Verschmutzungen in der Flüssigkeit, Verkrustungen oder Schaumbildung haben keinen Einfluss auf die Füllstandserfassung. Das Sondenmaterial kann entsprechend der geforderten chemischen Beständigkeit in Edelstahl (Werkstoff 1.4571), Edelstahl mit PFA-Überzug oder Edelstahl mit Tegumit-Überzug (WeichPVC) ausgeführt werden. Die Sondenlänge ist zwischen $100 \mathrm{~mm}$ und $2.500 \mathrm{~mm}$ frei wählbar. Zur einfachen Inbetriebnahme wird das Niveaumesssystem vorkonfiguriert ausgeliefert. Es ist kein Abgleich vor Ort mehr notwendig.

Tel. 09122 9855-0, www.mazurczak.de Halle 3, Stand C 65

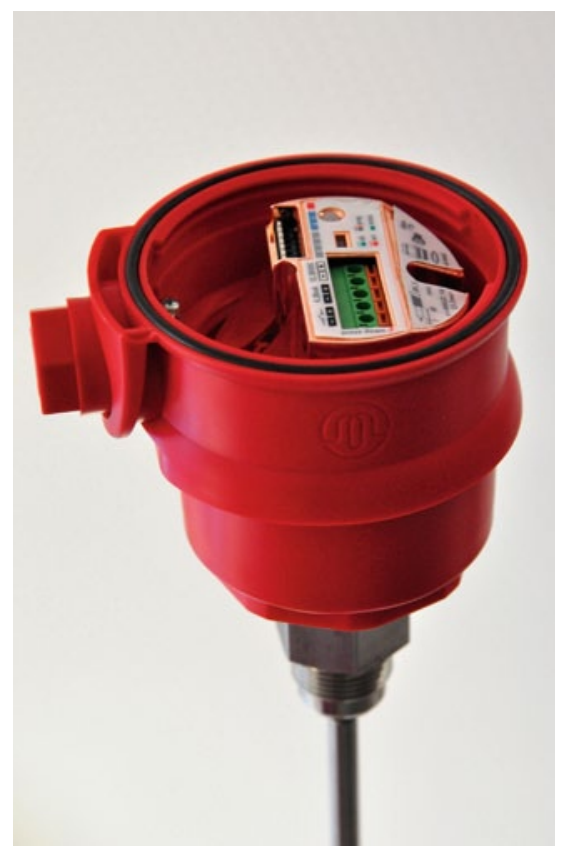

